\title{
A Peer Review of the Relationship of Socio-Economic Status on Contraction of Tuberculosis among Tb Patients in Windhoek District, Namibia: A Public Health Practice Perspective
}

\author{
Article by Francis Farai Chikuse \\ PhD Public Health Student of Texila America University, Namibia) \\ Email: francis.chikuse@gmail.com
}

Source: Murimba W. (2014). The Relationship of Socio-Economic Status on Contraction of Tuberculosis among TB Patients in Windhoek District, Namibia: A Public Health Practice Perspective. Online Journal of Medicine and Medical Science Research

http://www.onlineresearchjournals.org/JMMSR

\begin{abstract}
The article by Murimba Winnie titled "The Relationship of Socio-Economic Status on Contraction of Tuberculosis among TB Patients in Windhoek District, Namibia: A Public Health Practice Perspective" is reviewed. It is published in Online Journal of Medicine and Medical Science Research Volume 3, Issue 2 of 2014. The review engage the logical presentation of writing, follow of ideas, content and structure and its efficacy in tackling the risk factors of in-patients infected with tuberculosis. Socio-economic factors which predispose people to $T B$ diseases have been investigated before and therefore the review will focus on the relevancy, objective and accuracy of this article on this topic.
\end{abstract}

Keywords: Socio-Economic Status, Poverty, Katutura Intermediate Hospital, Tuberculosis, Poverty, Namibia.

\section{Article structure and summary}

The article presents an abstract which asserts that the study is a quantitative descriptive study. It was conducted among TB inpatients at Katutura Intermediate Hospital in Windhoek, between June and August 2011. Using a universal sampling method, all 38 TB inpatients were enrolled in the study. The socio-economic factors which predispose people to the transmission of TB infection among urban participants were identified and described. Corbett and colleagues (2009) postulate that the socially and economically disadvantaged urban poor are the most vulnerable to TB because of poor living standards, which enhance transmission of TB infection. The majority of the respondents in this article were residents of severely poverty stricken informal settlements in Windhoek District. The authors used some reputable sources, for example, TB case notification rates, incidence rates, treatment success rates and mortality rates to elucidate the magnitude of TB burden in Namibia. These statistics are derived from the Republic of Namibia, Ministry of Health and Social Services, World Health Organization (WHO) and World Bank reports.

Descriptive statistics, a table and a pie chart are used to present the study findings. Thereafter, interpretation and discussion of the results is done leading to a conclusion. The evidence based relationship between the socio-economic factors and TB infection are informative to the policies that tackle the risk factors which make people susceptible to TB infection among the urban poor. There are links to references, citation, subjects and author allowing the reader to easily review and evaluate the article. However, a thought consideration could have been given not only to the results of the study but also the rigor of the research to enhance its quality. Rigor refers to the extent to which the researchers worked to ensure the quality of the article. Issues of validity of the study could have been addressed extensively. For example, face validity could have been done where experts in the subject matter could have been asked whether the instrument measures the concepts intended. A data collecting instrument can 
DOI: $10.21522 / \mathrm{TIJPH} .2013 .06 .02 . A r t 020$

ISSN: $2520-3134$

be reliable but not valid. The article states that data was collected by five final year nursing students. There was no indication that prior training was provided to data collectors and how missing data was addressed during the collection and analysis stages.

The authors could have enumerated the incidence,prevalence and mortality rates of drug resistant TB among the inpatients. Recent reports by the Namibia, National Tuberculosis and Leprosy Programme avers that cases of drug resitance tuberculosis are increasing since 2007. For example, during 2015, 190 patients commenced treatment for MDR-TB, compared to 137 cases reported in 2014. Furthermore, three XDR-TB cases were notified, whereas, the number of cases of poly-drug resistance TB cases increased to seventeen in 2015 up from seven cases reported in 2014 (MOHSS, 2017). The unfavourable outcomes were stated as death (4\%), failure (4\%) and loss to follow up (4\%). For MDR-TB patients, the treatment success rate declined to $63 \% \%$ in 2013 down from $68 \%$ for the 2012 cohort. This illustrates that current control strategies have a weakness of containing the drug resistance tuberculosis epidemic. TB is disease closely linked to gender differences, poverty, unemployment and overcrowding. Globally, DR-TB is a continuing threat. In its 2017 annual report, WHO confirms that there were 600000 incident cases with rifampicin resistance tuberculosis (RRTB) in 2016, the most efficacious first-line regimen. Among these incident cases, 490000 has MDR-TB. MDR-TB is estimated to account for approximately $20 \%$ of TB mortality, with mortality rates approximated at $40 \%$ for MDR-TB patients and $60 \%$ among XDR-TB patients (WHO, 2015). The article could have documented the incidence, prevalence and mortality rates of DR-TB among study participants. Future studies should focus on comparison of age-standardized incidence, prevalence and mortality rates among DR-TB and drug resistance in other high burden diseases like malaria and HIV in Namibia. Other studies can compare socio-economic risk factors for TB and DRTB.

\section{Literature review}

TB is a major global health problem affecting many poor countries. According to WHO (2013), 22 high TB burden countries are developing countries where $25 \%$ of world' poorest population lives and this account for $80 \%$ of the global TB cases. David (2010) confirms that highest prevalence rates of TB are found among the productive age group of 20 to 55 years. This age group is at most risk for exposure to TB bacillus. Gender has also been implicated as a major risk factor for TB. Omara and colleagues (2012) reported gender disparities in TB cases, with highest prevalence rate of the disease among woman as compared to their male counter parts. Treatment for TB is free in most countries including Namibia and hence unemployment people have access to free TB diagnosis and treatment. Availability of financial resources often affects supportive measures to health seeking such as transport fees. David (2010) asserts that major factors that make people vulnerable to TB infection include poor housing, overcrowding, exposure to environmental pollution, personal habits like smoking and alcohol abuse. HIV co-infection has also been reported as a major predisposing factor. Globally, WHO (2015) reported a decrease in the TB incident cases over the past two decades in most parts of the world. However, the decrease in TB incidence rates is still lower than the mortality rates in sub-Saharan African region. The setting for this article is Windhoek Namibia and indeed the increase in TB mortality rates is attributable to co-infection with HIV. Namibia is ranked among top ten countries with highest HIV prevalence levels in world. WHO (2015) estimate adult HIV prevalence rate at $18.8 \%$.

\section{Article critique}

\section{Author}

The author Murimba Winnie and colleagues are lecturers in School of Nursing and Public Health Department of University of Namibia in Windhoek. This established the credibility of the authors. The article is written in a logical format highlighting paucity in literature of socio-economic factors which predispose people to TB infection. The language used throughout is clear with no ambiguities. The authors focused on ideas and therefore managed to set the stage for the problem they are reporting. 


\section{Accuracy}

To illustrate the burden of TB and HIV diseases in Namibia, the authors use credible and current sources like WHO and World Bank reports. List of all in-text sources is given at the end of the article. They used this information to justify why understanding risk factors of TB disease are important in advocacy and social mobilization efforts to support diagnosis and treatment programs. However, a closer analysis of the study method reveals a major limitation of a small sample size of 38 participants. Murimba and colleagues could have increased the target population by enrolling those TB patients attending the outpatient department but not hospitalized. The article makes use of passive case finding method. They could have included cases in the communities through active case finding of TB among household and neighboring contacts to increase the sample size. The authors acknowledged that due to small sample size the results cannot be generalized to all TB patients in Namibia. An explanation of the inclusion criteria for participation in the study is given and a pilot study was conducted to ensure reliability of the study.

\section{Relevance}

A quantitative descriptive research design are used to identify and describe the socio-economic risk factors for TB among inpatients. The article is meant to inform researchers and scholars and not entertain. This is an academic journal on a reputable academic data base. The authours managed to identify socioeconomic factors as gender, places of residence, exposure to enviromental pollution, employment status, nutritional status, social habits, HIV co-infection and knowledge about TB disease. One weakness of the method used is that analysis of data is mainly descriptive using frequencies and percentage distributions. Thus, the article failed to provide a statisitical test to show an association or relationship of these variables with TB disease. The title of the article is about the relationship of socio-economic status on contraction of TB. Therefore, a fair conclusion could have been that out of $38 \mathrm{~TB}$ inpatients the age range of respondents was 14-50 years, majority of them were from informal settlement who lived in shacks and were exposed to dust. Most of the respondents were HIV positive and were taking alocohol. Therefore, these factors could be drivers of TB infection among respondents.

\section{Objective}

The purpose for the study was to identify the risk factors of inpatient infected with TB at Katutura Intermediate Hospital in Windhoek District of Namibia. This objective was met. The objective of the study is clearly stated, limitations outlined and issues of ethical clearance are addressed. However, the data analysis plan could have been improved as it is mainly descriptive using frequency and percentage distributions. This is the major weakness of the study as the relationship between the risk factors and TB is difficult to establish from the given data. Parametric tests like analysis of variance (ANOVA) and Pearson correlation tests or non-parametric tests like Spearman correlation tests and Kruskal-Wallis test could have been employed to establish association and relationship between the risk factors and TB disease.

\section{Stability}

The article is published in Online Journal of Medicine and Medical Science Research journal. Online Journal of Medicine and Medical Science Research (ISSN 2277-0879) is a monthly open access, double blind peer reviewed, international and multidisciplinary journal.

\section{Analysis of graphs and tables}

The authors used one table and one pie chart to summarize the data according to gender and employment status respectively. A balance was struck between the text and the use of tables and graphs. 
DOI: $10.21522 / \mathrm{TIJPH} .2013 .06 .02 . A r t 020$

ISSN: $2520-3134$

\section{Advance knowledge on the topic}

TB diagnosis has been taken by the tides of innovation, with development of new molecular techniques for diagnosis of TB and DR-TB. WHO (2015) approved the use of Xpert MTB/RIF in 2010 and this assay transformed the field of TB diagnosis by offering sensitive and rapid diagnosis as well as detecting rifampicin resistance. According to WHO (2017), by 2016, more than 23 million Xpert MTB/RIF tests had been processed in 130 countries and MDR-TB detection has more than tripled. However, the major limitation of Xpert MTB/RIF is that its sensitivity is low in smear-negative and HIV associated TB. Currently new approaches are Xpert Ultra (Cephaid) and those targeted whole-genome sequencing are in the final stages of development. Ultra is a second-generation test that is designed to overcome sensitivity imperfect of Xpert MTB/RIF. The Xpert MTB/RIF Ultra assay was redesigned to boost analytical sensitivity more than ten times and improve reliability of detecting rifampicin resistance based mutations. Its sensitivity, accuracy and precision almost equals that of liquid culture based assays with an added advantage of being faster and user friendly at point of care.

At population level, Ultra is useful in detecting epidemics and monitoring drug surveillance. More work still needs to be done to explore potential utility of this test for public health intervention. On the treatment side, small studies indicate that treatment regimens that include Linezolid can be used to successfully treat MDR-TB and Sutezolid is also in phase II studies (Field et al., 2012). Preliminary evidence suggest that these drugs offer more hope as they have a satisfactory bioavailability and may be better tolerated by patients. Additionally, two fluoroquinolones moxifloxacin and gatifloxacin are currently in phase III clinical trials (Field et al., 2012).

\section{Conclusion}

The article by Murimba Winnie is admissible as it provides evidence that can be utilized to improve socio-economic status of marginalized people to support TB and HIV diagnosis and treatment. Improvement is socio-economic status removes barriers to treatment initiation. This calls for the strengthening of policies that integrate and intensify HIV and TB infections interventions into community health initiatives. Vitalizing the health systems for early detection of drug susceptible and DR-TB and furtherance in diagnosis, treatment and follow-up should therefore be the prime targets. Namibia has a higher burden of TB than predicted by her socio-demographic index and should enhance investigating reasons for this mismatch and appropriate solutions. Future studies can focus on risk factors for TB infection at community levels among close contacts and ways of addressing stigma and rehabilitations efforts among people with co-morbidities. Pilot studies can be done to establish the role of social protection programs in TB control arsenal. The content, structure, strengths sand limitations of the article were analyzed and critiqued.

\section{References}

[1].Corbett E.L., Bandason T., Cheung Y.B., Makamure B., Dauya E., Munyati S.S., Churchyard G.J., Williams B.G., Butterworth A.E., Mungofa S., Hayes R.J., Mason P.R (2009). Prevalent Infectious Tuberculosis in Harare, Zimbabwe: Burden, Risk Factors and Implications for Control. Int J Tuberculosis Lung Disease; 13(10): 1231-1237.

[2].David Y. (2010). An Examination of Known Tuberculosis Risk Factors and their Correlation across the United States. (Master's Thesis). USA: Georgia State University.

[3].Field S.K., Fisher D., Jarand J.M., Cowie R.L. (2012). New treatment options for multidrug-resistant tuberculosis. The Adv Respir Dis' 6: 255-268

[4].Omara F.D., Sarwat K.S., Abrar A.C., Ejaz Q. (2012). Gender disparity in tuberculosis cases in Eastern and western provinces of Pakistan. BMC Infect Diseases, 12(244).

[5].Republic of Namibia, Ministry of Health and Social Services (2017). National Tuberculosis and Leprosy Control Programme 2015/2016 Annual Report. MOHSS: Republic of Namibia.

[6].WHO (2011). Global Tuberculosis control: WHO Report. Geneva: World Health Organization.

[7].WHO (2015). Global Tuberculosis Report. Geneva: World Health Organization. 
Texila International Journal of Public Health

Volume 6, Issue 2, Jun 2018

[8].WHO (2017). Global Tuberculosis Report. Geneva: World Health Organization.

[9].World Health Organization website. WHO monitoring of Xpert MTB/RIF roll-out. Accessed January 302018.

http://www.who.int/tb/areas-of-work/laboratory/status_xpert_rollout_dec_2016.pdf. 